\title{
Regards
}

\section{Jean-Marie Legay ou la méthode des modèles}

\author{
Sylvie Lardon \\ Géographe, Inra \& AgroParisTech, UMR Métafort, 63170 Aubière, France
}

Jean-Marie Legay, agronome et biométricien, a été précurseur de l'interdisciplinarité dans les sciences de la vie en formalisant la méthode des modèles pour rendre compte de l'organisation des systèmes complexes ${ }^{1}$. Étudiante en biométrie à Lyon, j'ai fait sa connaissance en 1973. Cette rencontre a été décisive pour mon orientation vers la recherche. Pour moi, comme chercheure et comme enseignante, Jean-Marie Legay a été, constamment, un "modèle ", dans l'acceptation de la complexité des systèmes ${ }^{2}$, dans l'acception du modèle comme instrument ${ }^{3}$ et dans l'intuition d'une démarche de modélisation construite ${ }^{4}$.

\section{Un itinéraire de recherche}

Dans le sillon de Jean-Marie Legay, j'ai construit mon itinéraire de recherche sur la modélisation spatiale des dynamiques territoriales. Les jalons importants de cet itinéraire ont été balisés avec lui.

\section{De la thèse à I'HDR}

Il a été mon directeur de thèse $\mathrm{e}^{5}$; il fut aussi mon directeur $\mathrm{d}^{\prime} \mathrm{HDR}^{6}$. Il a inspiré mon parcours, en restant

Auteur correspondant : sylvie.lardon@agroparistech.fr

1 Voir aussi, dans ce numéro et le précédent, les autres hommages à Jean-Marie Legay.

2 Legay, J.-M., 1986. Méthodes et modèles dans l'étude des systèmes complexes, Cahiers de la recherche-développement,11,1-6.

3 Legay, J.-M., 1973. La méthode des modèles, état actuel de la méthode expérimentale, Informatique et Biosphère, 1, 5-73.

4 Legay, J.-M., 1997. L'expérience et le modèle. Un discours sur la méthode, Paris, Inra Éditions.

5 Lardon, S., 1980. Modèles de décision d'occupation du sol par les agriculteurs. Contribution à l'étude de quelques facteurs. Cas de la commune de Saint-Just Chaleyssin. Thèse de doctorat en sciences biologiques, Université Claude-Bernard Lyon 1.

6 Lardon, S., 1999. Modélisation spatiale des dynamiques territoriales. Mémoire d'habilitation à diriger des recherches en biométrie, Université Claude-Bernard Lyon 1. tout à la fois proche et lointain tout au long de ma progression. J'assume le choix que j'ai formalisé dans mon HDR :

«J'ai choisi d'utiliser la modélisation spatiale comme démarche de recherche, pour expérimenter, évaluer et généraliser des connaissances, parce qu'elle me fournit un cadre d'explication et d'interprétation. Les modèles de représentation de l'espace sont des outils de formalisation et de communication. Le langage graphique est universel, il permet le dialogue entre les disciplines et entre les chercheurs et les acteurs. La modélisation spatiale est aussi une démarche instrumentée pour aider les acteurs à raisonner des alternatives et à trouver des solutions innovantes. Les modèles spatiaux sont des outils de négociation et de résolution de problèmes. Les représentations spatiales servent d'objets médiateurs entre les acteurs, ils articulent différents points de vue et donnent à voir les différentes alternatives ${ }^{7}$. »

Jean-Marie Legay, évaluateur reconnu, sans concession mais toujours positif, a validé mon orientation, en reconnaissant mon engagement dans beaucoup d'animations, à mettre au crédit de la formation ${ }^{8}$.

\section{Des sciences de la vie aux sciences de la société}

Jean-Marie Legay a ouvert la voie, avec d'autres, de l'interdisciplinarité entre sciences de la vie et sciences de la société 9 . J'ai relaté mon itinéraire méthodologique en trois étapes, articulant modèles agronomiques,

\footnotetext{
7 Ibid.

8 Chadoeuf, J., Osty, P.L., 1999. Modéliser les transformations de l'espace par les activités agricoles et rurales. Comprendre les dynamiques territoriales. Aider à la conception de projets de gestion de l'espace, Supplément Sadoscope, Inra, SAD, 101, 4 pages.

9 Legay, J.-M. (Ed.), 2006. L'interdisciplinarité dans les sciences de la vie, Versailles, Quæ.
} 


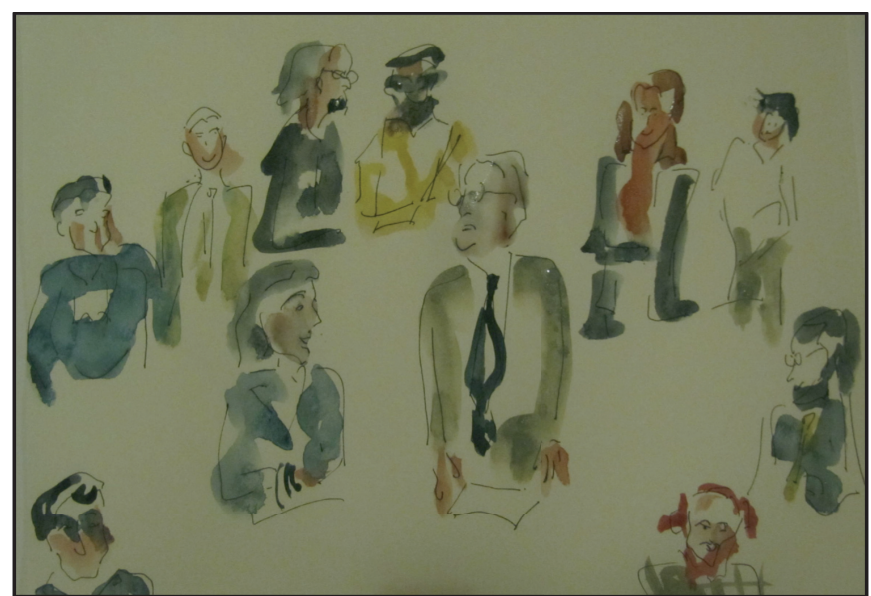

Fig. Aquarelle de Sylvie Lardon représentant les personnes présentes à la soutenance de son HDR (24 septembre 1999). Au centre, Jean-Marie Legay et Sylvie Lardon. En haut, de droite à gauche, les autres membres du jury : Christian Gautier (biométrie), Bernard Seguin (agronomie), Lena Sanders (géographie), Pascal Monestiez (biométrie), Jean-Pierre Deffontaines (agronomie), Alain Pavé (biométrie), Claude Millier (informatique). En bas à droite, Joël Chadoeuf (biométrie) et, en bas à gauche, Pierre-Louis Osty (agronomie) qui ont relaté cette soutenance dans le Sadoscope.

géographiques et informatiques ${ }^{10}$. La première étape consiste à aller chercher des concepts dans d'autres disciplines pour les adapter à une problématique spécifique. La seconde étape est la confrontation à d'autres points de vue, pour un enrichissement mutuel ou un approfondissement justifié. La troisième étape est la mise à l'épreuve des concepts et des méthodes dans des dispositifs adaptés de formation ou de conception collaborative.

J'en ai tiré une conclusion sur l'usage des modèles en interdisciplinarité :

«Dans les recherches interdisciplinaires, il faudrait donner aux modèles le rôle d'objets intermédiaires, appropriables par chaque discipline et transformés par chacune d'elle. La confrontation ne se ferait pas par opposition de concepts ou divergence de méthodes, mais par construction progressive d'artefacts qui, par leur consistance, obligerait à argumenter et à affiner les raisonnements. La modélisation serait alors l'invention d'un langage de formes, une sorte d'ingénierie de la connaissance s'appliquant à des objets modulables et modelables ${ }^{11}$. »

Jean-Marie Legay est le représentant de ce type de modélisation.

10 Lardon, S., 2006. Modélisation spatiale et approche agronomique, in Legay, J.-M. (Ed.), L'interdisciplinarité dans les sciences de la vie, Versailles, Quæ, 85-102.

11 Ibid.

\section{Des représentations spatiales à la gouvernance du changement}

Jean-Marie Legay était aussi sensible à l'engagement $\mathrm{du}$ chercheur dans l'action. Dans mon dernier dossier d'évaluation en 2012, je conclus sur mon itinéraire de recherche :

« Je me situe dans une posture de recherche constructiviste et systémique. La vision prospective s'avère d'actualité, même si elle a été posée sur une intuition plus que sur une théorie. Le temps de la recherche rejoint ainsi celui de l'action, en étant au cœur des questionnements des acteurs des territoires et en les accompagnant par la formation, avec les concepts, méthodes, outils et dispositifs de la recherche. À l'heure où l'évaluation des chercheurs reconnaît enfin la pluridisciplinarité et la formation comme critères d'évaluation, il importe de valoriser cette expérience et ce savoir-faire, pour faire évoluer les pratiques des institutions. »

Je contribue à formaliser les activités de conception des chercheurs qui accompagnent les changements dans les territoires ruraux et périurbains. Les démarches partenariales mises en œuvre et l'interdisciplinarité vécue sont les garantes d'une approche inscrite dans l'action. La rigueur scientifique des itinéraires méthodologiques construits et la diversité assumée permettent de monter en généricité. C'est cela que Jean-Marie Legay m'a appris.

\section{Un chemin partagé}

Jean-Marie Legay était un personnage sobre et discret, il se tenait en retrait des relations humaines, peutêtre parce qu'il était justement un humaniste. J'ai pu partager avec lui des moments de connivence, je relate pour vous quelques anecdotes de mon parcours.

\section{Sur la voie de la recherche}

Quand Jean-Marie Legay fait son premier cours, dans un amphithéâtre de l'Université Claude-Bernard de Lyon, à l'automne 1973, alors que je commence une formation en Deug de biologie, je comprends que j'ai trouvé là ce que je cherchais. J'y suis encore, et si c'était à refaire, je le referais de la même façon! Il était tout de même étrange de rencontrer un laboratoire de biométrie, faisant se côtoyer la biologie et les mathématiques, au sein d'une université des sciences du vivant. Et pourtant, ce laboratoire existait et était très actif. Que d'heures agréables, passées sur les paillasses, à élever des drosophiles ou à ensemencer des boîtes de Pétri, à suivre les courbes logistiques ou à composer des modèles à compartiments!

L'été 1977, celui de mon DEA, je travaille au laboratoire et m'occupe de l'élevage des Bombyx mori, support d'expérimentation. Je marche ainsi dans les traces de 
Jean-Marie Legay, qui avait démarré sa carrière à Alès, chargé de l'amélioration de la sériciculture et de la sélection du ver à soie. Paillasses et chambres froides ont été le lieu de formulation de mon désir de m'orienter dans la recherche.

Plus tard, durant mon doctorat, je l'accompagne à Alès - lieu lié pour moi à l'imaginaire du ver à soie pour rencontrer sur le terrain du PIREN CaussesCévennes coordonné par Marcel Jollivet ${ }^{12}$, mes futurs collègues de l'Inra-SAD. L'ouverture interdisciplinaire était en acte. Olivier Dollfus, géographe spécialiste des Andes, me fait soutenir mon oral de DEA sur les modèles spatiaux en géographie, dans un gîte au col du Lautaret, au Monêtier-les-Bains, où se situait le « siège » des activités de recherche DGRST sur le Briançonnais ${ }^{13}$, au milieu des étudiants venus faire du terrain.

Les rencontres avec Jean-Marie Legay étaient toujours constructives pour une jeune doctorante. J'en garde le souvenir de moments intenses et sereins, y compris à la maison de campagne de la famille Legay, dans la commune de Saint-Just Chaleyssin, lieu de mon terrain de thèse sur les modèles d'occupation du sol par les agriculteurs.

\section{De la biométrie à l'agronomie et la géographie}

Mais Lyon m'ennuie. Je pars à Grenoble, tout en restant monitrice de TD de mathématiques à l'Université Claude-Bernard. Jean-Marie Legay m'accompagne au laboratoire d'économie de Grenoble, car il veut que je $m$ 'insère dans un milieu scientifique. Je fuis l'économie aussi vite que je suis arrivée, pour me ressourcer... en géographie. Ce sera le début d'une collaboration scientifique et amicale en géographie, avec Bernard Debarbieux ${ }^{14}$, actuellement professeur d'aménagement du territoire à Genève.

Je pars en coopération en Algérie avec le projet de monter un laboratoire de biométrie sur le modèle de

\footnotetext{
12 Programme PIREN : Programme de recherche en environnement financé par le CNRS, faisant explicitement appel aux approches pluridisciplinaires pour comprendre le fonctionnement économique, social et technique d'un territoire. $C f$. Jollivet, M., 1984. Réflexions méthodologiques sur le programme Causses-Cévennes, Paris, CNRS/PIREN.

13 DGRST : Délégation générale à la recherche scientifique et technique. Première structure de recherche scientifique et technique concrétisant la présence des sciences sociales dans les dispositifs de politiques publiques. Cf. Dollfus, O. et al., 1981. Recherches en Briançonnais, Grenoble, Cemagref.

${ }^{14}$ Ses recherches portent sur les imaginaires et les formes de la connaissance géographiques et de la gestion de l'environnement de l'aménagement. Il a dirigé un groupe de prospective à la DATAR (Paris) sur "Représentations et complexité territoriale ». $C f$. Debarbieux, B., Lardon, S., 2003. Les figures du projet territorial, La Tour d'Aigues, Éditions de l'Aube.
}

celui de Lyon, sous la direction du professeur Nigon, avec deux autres collègues, un professeur et un assistant, étant moi-même maître-assistante. Le projet de laboratoire ne s'est pas réalisé, mais c'est là que je fais mes débuts dans l'enseignement, avec un amphithéâtre bourré d'étudiants (la plupart étant des hommes plus âgés que moi). J'y enseigne la génétique et l'informatique «papier-crayon», car il n'y a pas d'ordinateurs en ce temps-là en Algérie. Jean-Marie Legay me rend visite à Oran, pour conforter ma contribution à la modélisation.

Après trois années de coopération, au retour, le ministère de la Coopération finance six mois de réinsertion pour ses chercheurs. C'est avec Jean-Pierre Deffontaines et Marc Benoit, chercheurs à l'Inra-SAD que je prends langue. De l'avis de Jean-Marie Legay : « il faut absolument que vous travailliez avec Jean-Pierre Deffontaines, c'est ce qui vous convient, parce qu'il a une approche globale de l'agriculture et du territoire, par le paysage ». Convenons qu'il avait vu juste, tant ce compagnonnage a été structurant pour la suite de mes recherches, d'une première rencontre à l'automne 1983, jusqu'à l'ouvrage publié récemment sur les traces de Jean-Pierre Deffontaines ${ }^{15}$.

\section{De l'agronomie et la géographie à l'aménagement des territoires}

J'entre ensuite, en 1986, dans le giron d'une grande maison, $\mathrm{l}^{\prime}$ Inra, au département $\mathrm{SAD}^{16}$. Une réunion initiale à Toulouse cadre mon insertion dans l'équipe, c'est bien de modélisation spatiale (terme alors inusité, du moins à l'Inra, qu'il a fallu défendre) dont il s'agira et pas seulement de modélisation systémique. C'estJean-Marie Legay qui sera là à ce début, comme plus tard lors de ma mutation à la Maison de la Géographie en 1994. Je prends mes distances vis-à-vis de la biométrie, et dans le rapprochement avec les systèmes techniques de PierreLouis Osty, agronome avec lequel j'ai travaillé sur le Causse Méjan ${ }^{17}$, je poursuis mes avancées et projets sur $l^{\prime}$ approche spatiale en agronomie des territoires ${ }^{18}$.

\footnotetext{
15 Lardon, S. (Ed.), 2012. Géoagronomie, paysage et projets de territoire. Sur les traces de Jean-Pierre Deffontaines. Versailles, Quæ.

16 SAD, Systèmes agraires et développement, devenu Sciences pour l'action et le développement.

17 Le Causse Méjan était l'un des terrains du PIREN CaussesCévennes et a été le lieu de différents projets de recherche. $C f$. Chassany, J.-P., Crosnier, C., 2009. Les grands Causses, terre d'expériences, Parc national des Cévennes.

18 Lardon, S., Deffontaines, J.P., Osty, P.L., 2001. Pour une agronomie du territoire: prendre en compte l'espace pour accompagner le changement technique. Les entretiens du Pradel, $1^{\text {ere }}$ édition, "Autour d'Olivier de Serres : pratiques agricoles et pensées agronomiques », partie agronomique, C.R. Acad. Agri, 4, 187-192.
} 
Par la suite, les relations avec Jean-Marie Legay se feront de loin en loin, dans diverses instances scientifiques, comme le colloque en 1997 sur « Les temps de l'environnement » où j'ai choisi de soutenir une HDR en biométrie avec Jean-Marie Legay, et non en géographie avec François Durand-Dastès ${ }^{19}$, pour faire reconnaître mes apports en modélisation spatiale pour le développement territorial.

Jean-Marie Legay sera à l'origine des liens que je développerai avec le laboratoire informatique nouvellement créé à l'Inra en 1988 par Claude Millier, qui luimême me fera rencontrer Vincent Piveteau, alors directeur du centre Engref (maintenant AgroParisTech) de Clermont-Ferrand. C'est avec ce dernier que nous impulserons les travaux de Recherche-Formation-Action en ingénierie du développement durable des territoires ${ }^{20}$, que je poursuis actuellement au sein de l'UMR Métafort à Clermont-Ferrand. Mais ça, c'est une autre histoire.

\section{Un fil de vie}

Il me reste des acquis de ma formation initiale en biométrie, sur lesquels je m'appuie toujours, trente-cinq ans après, car c'est au fondement de ma démarche. J'enseigne à mon tour aux étudiants de master la "méthode des modèles ». C'est ce qui assure la rigueur scientifique de mon approche non conventionnelle de recherche-action en lien avec les questions de société, auxquelles Jean-Marie Legay m'a ouverte, dans les grandes idées comme dans les petits détails.

Jean-Marie Legay a été, pour moi, un fil conducteur, un fil de vie.

\footnotetext{
19 Je travaillais alors avec le laboratoire Géographie-cités, dans un programme de recherche européen Archaeomedes II (19961999) : " Policy-relevant models of the natural and anthropogenic dynamics of degradation and desertification and their spatiotemporal manifestations » (Program Environment of the European Commission DGXII), coordination S. van der Leeuw (Arizona State University).

${ }^{20}$ Lardon, S., Piveteau, V., 2005. Méthodologie de diagnostic pour le projet de territoire : une approche par les modèles spatiaux, Géocarrefour, 80, 2, 75-90.
} 